had no effect on incidence. Most JMT backpackers follow standard backcountry hygiene recommendations.

Conclusions.-JMT backpackers have comparatively lower risk of experiencing diarrhea than other major long-distance backpacking routes in the United States. Diarrhea incidence is unaffected by backpacker demographics or hygiene compliance regularity on the JMT.

\section{Iloprost With and Without rt-PA: Treatment of 131 Cases of Severe Frostbite}

Emmanuel Cauchy ${ }^{1}$; Eric Chetaille ${ }^{1}$; Emmanuel Pham ${ }^{1}$;

Hugo Nespoulet ${ }^{1}$; Pascal Zellner ${ }^{1}$; François Becker ${ }^{1}$; Peter Hackett ${ }^{2}$

${ }^{1}$ IFREMMONT (Institut de Formation et de Recherche en Médecine de Montagne), Hôpital de Chamonix, Chamonix, France, ${ }^{2}$ Institute for Altitude Medicine, Telluride, CO, USA

Introduction.-Both rt-PA and iloprost (prostacyclin) are effective treatments for severe frostbite, but it is unclear if using them in combination is more effective.

Objective.-To evaluate combined treatment with rt-PA and iloprost in severe frostbite, compared with other therapies.

Methods.-Retrospective case review of 131 patients with severe frostbite. Patients without contraindication or severe trauma were managed with rapid rewarming $\left(38^{\circ} \mathrm{C}\right.$ bath water immersion + aspirin IV [250mg] + buflomedil IV [400 mg, alphalytic vasodilator] during 1 hour. Then 41 patients received daily treatment of aspirin and buflomedil (TT-A); 58 received aspirin and IV iloprost 2 $\mathrm{ng} / 6 \mathrm{~h}$ (prostacyclin) (TT-B); and 20 received aspirin, IV tPA (100 mg, first day only) and iloprost (TT-C). Twelve patients did not receive any treatment (TT-D). The final level of amputation was measured 3 to 8 days later on bone scanning.

Results. - For grade 3 frostbite, $100 \%$ of the patients required amputation in TT-D (4 of 4 patients), as did $62.5 \%$ with TT-A (10 of 16 patients), $4.9 \%$ with TT-B ( 2 of 41 patients) $(P<.01)$, and $27.3 \%$ with TT-C ( 3 of 11 patients) $(P<$ $.03)$. For grade $4,100 \%$ needed amputation in TT-D ( 3 of 3 patients), $100 \%$ in TT-A ( 4 of 4 patients), $66.7 \%$ TT-B $(4$ of 6 patients $)(P<.001)$ and $44.4 \%$ with TT-C ( 4 of 9 patients) $(P<0.03)$. Of 407 digits, 183 frozen fingers lead to 22 amputations (TT-A 49\%, TT-B 0\%, TT-C 2\%, TT-D 49\%), and 224 frozen toes lead to 25 amputations (TT-A 34\%, TT-B 0\%; TT-C 4\%, TT-D 62\%). Analysis showed that tPA was effective up to 12 hours and iloprost up to 48 hours.

Conclusion.-The efficacy of iloprost was significantly higher than buflomedil or no treatment. The addition of rt-PA to prostacycline seems to improve the prognosis only of stage 4 frostbite and only when the delay between rewarming and treatment was less than 12 hours.

Search and Rescue (SAR) and Fatalities in Mount Rainier National Park (MORA) 2010-2015: Location and Altitude Data May Enhance SAR Training and Preventative Search and Rescue (PSAR)

Brian Scheele

Mount Sinai Medical Center, Miami Beach, FL, USA

Introduction.-Visits to our national parks number in the millions each year. Despite efforts by search and rescue (SAR) personnel to aid visitors, fatalities do occur. Mount Rainier National Park (MORA) encompasses 236,381 acres $\left(956.60 \mathrm{~km}^{2}\right.$ [369.35 miles$\left.\left.{ }^{2}\right]\right)$ and is centered around the stratovolcano Mount Rainier (4,392 m [14,411 feet]).

Objectives.-To provide an initial source of reference in the medical literature describing publicly available data on SAR and fatalities in MORA and to quantify location and altitude data regarding fatalities that required SAR activation in MORA to enhance SAR preparedness and PSAR activity.

Methods.-A retrospective review of a National Park Service publicly available database.

Results.-Between January 2010 and September 2015, 239 SARs and 41 fatalities occurred in MORA. Fatalities occurred in 22 defined locations, with 3 fatality locations unknown. Not all fatalities involved SAR. Thirty-two fatalities (78\%) were related to activity of 24 SARs $(10 \%)$, and 9 fatalities $(12 \%)$ were unrelated to SAR activity. Location of the 32 SAR-related fatalities was concentrated in 18 defined locations with 2 fatality locations unknown. Seven locations had more than one SAR-related fatality, yet only 2 individual SARs were related to more than one fatality. Thirteen SAR-related fatalities occurred at very high altitude ( $>3500 \mathrm{~m}[>11482$ feet]), 12 at high altitude $(1500-3500 \mathrm{~m}$ [4921-11,482 feet]), and 7 at low altitude ( $<1500 \mathrm{~m}[<4921$ feet]). SAR-related fatalities occurred at a mean elevation of $2645 \mathrm{~m}$ (8679 feet), SD $1225 \mathrm{~m}(4022$ feet), and interquartiles $1584 \mathrm{~m}, 2853 \mathrm{~m}$, and $3825 \mathrm{~m}$ (Q1 5200 feet, Q2 9362 feet, Q3 12,550 feet).

Conclusions.-Between 2010 and 2015, 10\% of SAR operations within MORA involved fatalities, and $78 \%$ of these SARs were located at high or very high altitude. These SAR operations and fatalities were located in distinct areas within MORA. SAR training and PSAR operations may benefit from quantified location and altitude-specific data.

Epidemiology of Mountain Climbing Injuries Presenting to Emergency Departments in the United States from 2012 to 2014

Benjamin Nicholson; Jacob Kallenberg; Jolion McGreevy

Boston Medical Center, Boston, MA, USA

Introduction.-There is currently a paucity of literature describing the burden and severity of mountain climbing-related injuries presenting to the emergency department.

Objective.-To determine the epidemiology of mountain climbing injuries presenting to US emergency departments.

Methods. - The Consumer Product Safety Commission's National Electronic Injury Surveillance System (NEISS) is a weighted database that provides a national probability sample of participating hospitals and allows estimation of product-related injuries treated in emergency departments in the United States. The NEISS database was queried for the period January 1 , 2012 through December 31, 2014 for product code 1258: "Mountain climbing (activity, apparel or equipment)." The results were analyzed for diagnosis, sex, race, anatomic location of the injury, size of the hospital, and disposition.

Results.-The NEISS database provided 367 representative unique cases, resulting in an estimated 14,147 visits nationally during the 3-year period. The patients were predominantly male $(65.0 \%)$ and white $(72.5 \%)$, with an average age of 27.6 years. The largest percentage, $34.8 \%$ (4922), was seen in small hospitals. Disposition from the ED was discharge in $88.3 \%$, admission in $7.7 \%$, and transfer to another facility in $3.2 \%$. The most common diagnoses were fracture $(4008,28.3 \%)$, strain or sprain $(3574,25.3 \%)$, and contusions/abrasions $(1658,11.7 \%)$. Internal organ injury $(611,4.3 \%)$ was less common. The lower extremities were commonly injured, including the ankle $(2909,20.6 \%)$, foot $(1553,11.0 \%)$, and lower leg $(1361,9.6 \%)$. The head $(1496,10.6 \%)$ was also a frequently injured body site.

Conclusions.-Musculoskeletal injuries commonly were observed in association with products coded as mountain-climbing related. The majority was discharged from the emergency department. Injuries predominately involved the lower extremities; however, $10.6 \%$ of patients had an injury to the head. Preventive strategies should focus on lower extremity protection and encourage the use of helmets.

Epidemiology of Scuba Diving-Related Injuries Presenting to the Emergency Department Between 2012 and 2014

Benjamin Nicholson; Jolion McGreevy

Boston Medical Center, Boston, Massachusetts, USA

Introduction.-Scuba diving injuries not reported to the Diver Alert Network are poorly described in the literature and the current burden of injury is unknown.

Objective.-To describe injury patterns observed nationally and influence future efforts aimed at developing countermeasures and training to better address the current burden of injury among scuba divers.

Methods. - The Consumer Product Safety Commission's National Electronic Injury Surveillance System (NEISS) is a weighted database that provides a national probability sample of participating hospitals that allows for estimation of product-related injuries treated in emergency departments in the United States. 